\title{
Age differences in arterial and venous extra-cerebral blood flow in healthy adults: contributions of vascular risk factors and genetic variants
}

\author{
Naftali Raz ${ }^{1,2} \cdot$ Ana M. Daugherty ${ }^{1,3} \cdot$ Sean K. Sethi ${ }^{4} \cdot$ Muzamil Arshad $^{1,5}$. \\ E. Mark Haacke ${ }^{4,6}$
}

Received: 18 March 2016 / Accepted: 24 December 2016 / Published online: 24 January 2017

(C) Springer-Verlag Berlin Heidelberg 2017

\begin{abstract}
Sufficient cerebral blood flow (CBF) and venous drainage are critical for normal brain function, and their alterations can affect brain aging. However, to date, most studies focused on arterial CBF (inflow) with little attention paid to the age differences in venous outflow. We measured extra-cerebral arterial and venous blood flow rates with phase-contrast MRI and assessed the influence of vascular risk factors and genetic polymorphisms ( $A C E$ insertion/deletion, COMT val158met, and APOE\&4) in 73 adults (age 18-74 years). Advanced age, elevated vascular risk, $A C E$ Deletion, and COMT met alleles were linked to lower in- and outflow, with no effects of $A P O E \varepsilon 4$ noted. Lower age-related CBF rate was unrelated to brain volume and was observed only in val homozygotes of COMTval158 met. Thus, in a disease-free population, age differences
\end{abstract}

Electronic supplementary material The online version of this article (doi:10.1007/s00429-016-1362-2) contains supplementary material, which is available to authorized users.

Naftali Raz

nraz@wayne.edu

1 Institute of Gerontology, Wayne State University, 87 E Ferry St. 226 Knapp Bldg., Detroit, MI 48202, USA

2 Department of Psychology, Wayne State University, 5057 Woodward Ave., Detroit, MI 48202, USA

3 Beckman Institute for Advanced Science and Technology, University of Illinois Urbana-Champaign, $405 \mathrm{~N}$ Matthews Ave., Urbana, IL 61801, USA

4 The MRI Institute of Biomedical Research, 440 E Ferry St., Detroit, MI 48202, USA

5 Department of Psychiatry and Behavioral Sciences, Wayne State University, 3990 John R, Detroit, MI 48201, USA

6 Department of Radiology, Wayne State University, 3990 John R, Detroit, MI 48201, USA in CBF may be notable only in persons with high vascular risk and carriers of genetic variants associated with vasoconstriction and lower dopamine availability. It remains to be established if treatments targeting alleviation of the mutable factors can improve the course of cerebrovascular aging in spite of the immutable genetic influence.

Keywords Jugular vein · Internal carotid artery · Vertebral artery $\cdot$ Phase-contrast MRI $\cdot C O M T \cdot A C E$

\section{Introduction}

The fact that the brain draws more than $20 \%$ of the body's blood supply while comprising only $2 \%$ of the total body weight (Attwell and Laughlin 2001) underscores the importance of studying cerebral blood flow (CBF). Investigating factors that influence $\mathrm{CBF}$ is particularly important in the context of brain aging. Several investigations have demonstrated with various in vivo methods that compared to young adults, healthy elderly have lower arterial CBF (Chen et al. 2011; Henriksen et al. 2014; Parkes et al. 2004; Shirahata et al. 1985; van Es et al. 2010; Zarrinkoob et al. 2015; Zhao et al. 2007), and a longitudinal investigation found arterial CBF declines over time (ten Dam et al. 2007).

Despite these descriptive findings, the mechanisms of age differences in CBF remain unclear. Age-related differences in the cerebrovascular system appear plausible candidates for explaining the reportedly diminished CBF in older adults. Advanced age is accompanied by progressively increasing risk for cardiovascular disease with prevalence, severity and cumulative detrimental effects of major vascular risk factors-such as hypertension, reduced cardiac output, obesity, sedentary lifestyle, and tobacco smoking 
(Mozaffarian et al. 2015)—steadily rising with advanced age. Indeed, in some extant studies, assorted vascular risk factors have been linked to lower arterial CBF and reduced cerebral perfusion beyond the influence of calendar age (ten Dam et al. 2007; Vernooij et al. 2008). Moreover, some findings suggest that although larger brains draw greater CBF (van Es et al. 2010; Vernooij et al. 2008; Zarrinkoob et al. 2015), age-related declines in CBF may exceed the magnitude expected from age-related parenchymal shrinkage (Chen et al. 2011).

Whereas arterial CBF in aging is relatively well described, significantly less is known about the venous drainage system that guides deoxygenated blood into the dural sinuses and then into the internal jugular veins (Doepp et al. 2004). The extant studies of age differences in brain drainage reveal slower internal jugular vein (IJV) flow and greater IJV lumen area (Chung et al. 2010; Magnano et al. 2016) with advanced age, and some have theorized that abnormalities of venous drainage might contribute to cerebral aging and cognitive declines (Zivadinov and Chung 2013). Thus, understanding the influences that shape CBF in both directions may elucidate structural and functional changes in the aging brain and show the way to possible mitigation thereof.

Blood flow to and from the brain is determined by multiple biomechanical, neurochemical, and physiological variables, and variations in CBF rate depend on blood viscosity, compliance of the blood vessels, structural remodeling of vascular beds as well as the levels and activity of multiple hormones and neurotransmitters that act as vasoconstrictors or vasodilators (Armulik et al. 2011; Casey et al. 2015; Hall et al. 2014; Henriksen et al. 2014; Nishijima et al. 2015). Dilation and constriction of the cerebral arteries and veins have a substantial effect on CBF (Armulik et al. 2011; Hall et al. 2014), and common genetic variants associated with vascular function may affect cerebral and extracerebral blood flow. Recent studies showed that motility of specialized contractile cells, and pericytes and contractility of the blood vessels are lower in carriers of a variant of the Apolipoprotein E gene, APOE\&4 (Casey et al. 2015; Hajjar et al. 2015). Although APOE\&4 is arguably the best known genetic risk factor for Alzheimer's disease (AD), it is also important in many vascular processes and the effects of its risky allele extend beyond AD (Song et al. 2004). Notably, $\mathrm{CBF}$ is lower in APOE $\varepsilon 4$ carriers regardless of AD diagnosis (Kim et al. 2013; Thambisetty et al. 2010). In addition, $A P O E \& 4$ variant has been linked to venous thrombosis and flow abnormalities in lower extremities (Zhu et al. 2014), although at the time of this writing, its effect on cerebral outflow is not known.

Compliance of blood vessels is also affected by several hormones and neurotransmitters that act as vasoconstrictors or vasodilators, such as angiotensin II, bradykinin, norepinephrine, and dopamine (Greenfield and Tindall 1968; Messina et al. 1975). Availability of a potent endogenous vasoconstrictor, angiotensin II, is controlled by the angiotensin converting enzyme ACE (also known as dipeptidyl-carboxypeptidase I), which in addition to enabling its synthesis from angiotensin I inhibits vasodilator bradykinin (Vauquelin et al. 2002; von Bohlen und Halbach and Albrecht 2006). Notably, a higher concentration of ACE in the cerebro-spinal fluid has been linked to lower CBF (Janciauskiene et al. 2009). As is the case with many enzymes, ACE is under genetic control and the $A C E$ gene that affects its production has a common functional polymorphism that is due to insertion (I) and deletion (D) of an Alu repetitive element in the intron of $A C E$. The change produces three genotypes: $\mathrm{Alu}^{+/+}, \mathrm{Alu}^{+/-}, \mathrm{Alu}^{--}$, or $A C E \mathrm{I} / \mathrm{I}$, $A C E \mathrm{I} / \mathrm{D}$, and $A C E \mathrm{D} / \mathrm{D}$, respectively. The insertion allele is associated with dose-related reduction in ACE activity and by thus limiting synthesis of angiotensin II, exerts influence similar to that of an ACE inhibitor-i.e., limits vasoconstriction. Carriers of the D (high activity) allele evidence increased susceptibility to vascular disease (Lao et al. 2005), increased risk for hypertension (Bautistaet al. 2008), increased risk for stroke of large vessels (Saidi et al. 2009), and greater leukoaraiotic burden in the brain (Hassan et al. 2002). In some studies, ACE D/D homozygotes are less prevalent in older age groups, indicating a possible negative impact on survival (Morris et al. 1994). Carrying ACE I (low activity) allele has been associated with lesser arterial stiffness (Benetos et al. 1996; Mattace-Raso et al. 2004; Taniwaki et al. 1999), although in some samples, the effects are sex-specific (Lynch et al. 2007). At the time of this writing, there are no studies that examine ACE I/D differences in arterial or venous CBF in healthy adults.

An influential vasoactive hormone, dopamine, exerts its influence as a vasodilator via its effect on the smooth muscles of the blood vessels (Zeng et al. 2007). Dopamine availability is regulated by multiple means, including pre- and postsynaptic receptors and catabolizing enzymes, such as catechol- $O$-methyl transferase (COMT). The activity of COMT is controlled by an eponymous gene COMT that has a common single nucleotide polymorphism (SNP) COMT val158met. The mutant met allele of that polymorphism is associated with fourfold reduced activity of COMT and thereby greater availability of dopamine, which plays an important role in regulating arterial blood pressure (Jose et al. 2003). In adults, COMT val/val genotype has been linked to systolic hypertension (Hagen et al. 2007) increased rate of conversion to dementia (Dixon et al. 2014), and in children, resting CBF is reduced with increased dose of the val allele (Thomason et al. 2009). There is, however, no comparable study of the influence that SNP may exert on cerebrovascular function in adults. Because the dopaminergic system undergoes significant 
and possibly nonlinear changes with age (Morgan et al. 1987), it is plausible that genetic propensity for higher dopamine availability may indirectly affect its correlates, including $\mathrm{CBF}$, in an age-dependent manner.

Given the effect common vascular risk factors have on brain aging (Jagust 2013; Raz and Rodrigue 2006), and the calls to pay more attention to disentangling contributions of calendar age and accompanying vascular changes (e.g., Morra et al. 2013), it is surprising that the effects of genetic variants on $\mathrm{CBF}$ have not before been evaluated in conjunction with the influence of common age-related vascular risk factors, such as hypertension. The aim of this study was, therefore, to examine the effects of common vascular risk factors and two genetic variants known to affect brain vascular function on CBF inflow and outflow. We hypothesized that in addition to older adults having slower blood flow rate, carriers of $A P O E \varepsilon 4$, and homozygotes for $A C E$ D or COMT val158 alleles would evidence additional reduction in arterial and venous blood flow.

\section{Method}

\section{Participants}

The sample was drawn from adult volunteers $(N=73$, 45 women, age 18-74 years), who took part in an ongoing longitudinal study and were recruited from the Detroit metropolitan area through advertisement in local printed and electronic media. Because blood flow assessment was introduced after commencement of the study and terminated before its conclusion, only a limited number of participants were recruited into this sub-study, based on their scheduled visits within a defined period, on a first-come, first-assessed basis. Thus, in the context of a larger study, this sample was not selected on any particular additional criteria and participants who were not included can be considered missing at random. The participants underwent phone interviews and completed a 66-item health questionnaire, from which information pertinent to their inclusion in the study was drawn. The reasons for exclusion were reported history of cardiovascular, neurological, and psychiatric conditions, head trauma with a loss of consciousness for more than $5 \mathrm{~min}$, history of alcohol and drug abuse, thyroid problems, hypertension, and diabetes. The items used to screen for cardiovascular disease included any sort of "heart troubles" and cardiovascular complaints as well as taking specific medications prescribed for treatment of cardiovascular symptoms. The participants had corrected visual acuity of 50/20 or better (Optec 2000, Stereo Optical, Chicago, IL) and hearing of $40 \mathrm{~dB}$ or better for frequencies of 500-4000 Hz (Maico, MA27, Eden Prairie, $\mathrm{MN}$ ). To screen for dementia and depression, we used the Mini-Mental State Examination (MMSE; Folstein et al. 1975) and the Center for Epidemiologic Studies Depression Scale (CES-D; Radloff 1977). Only persons who scored 26 or above on the MMSE and 15 or below on the CES-D were invited to participate. All participants provided written informed consent in accord with university and hospital review board guidelines. Because the phase-contrast MRI sequence was introduced late in the study, the participants were at various stages of longitudinal assessment and insufficient cognitive data common to all participants across the stages of study did not allow for testing cognitive correlates.

\section{Vascular risk assessment}

We measured blood pressure on four separate days by a mercury sphygmomanometer (BMS 12-S25) with a standard blood pressure cuff (Omron Professional) on the left arm, with participants seated in a comfortable chair. The systolic and diastolic measures were averaged for each individual across sessions. The data on exercise regimen, smoking, and history of hypertension as well as anti-hypertension medication were obtained through a questionnaire. A compound vascular risk index was designed to reflect recognized factors for cardiovascular disease (Mozaffarian et al. 2015), and was computed as VR $=$ Hypertension $+((($ Sys tolic Blood Pressure - 120)/20)) + (BMI/20) + Smoking + Exercise $+($ Exercise Frequency/7) $)$, where hypertension diagnosis (8 participants reported), history of smoking (5 participants reported), and reported engagement in physical exercise were dichotomous incidence variables ( 0 vs. 1 , with 14 participants reporting no exercise-like physical activity); exercise frequency was the number of days exercising per week; BMI, body-mass index was computed as weight over squared height $\left(\mathrm{kg} / \mathrm{m}^{2}\right)$ and scaled by 20 ; and the systolic blood pressure measure was an average computed over 2-4 (most commonly 4) occasions, referenced against the normative value of $120 \mathrm{mmHg}$ and scaled by 20 to bring it to the common scale and avoid undue influence on the composite score.

\section{Blood panel data}

Hematocrit determines blood viscosity and therefore affects blood flow. In addition, older persons may have lower hematocrit (Aanerud et al. 2012; Henriksen et al. 2014); we examined if this was the case in our sample. We found that although, as expected, men had higher hematocrit than women [ 44.0 vs. $40.35 \%, F(1,61)=32.158, p<.001]$, within the normal range of hematocrit values sampled here, no differences were noted with respect to age, vascular risk, or genetic variants (all $F \leq 1$ ). As there was no association between hematocrit with age or blood flow rate $(r=-.02$ 
to $0.08, \mathrm{~ns}$ ), we did not add hematocrit to the statistical model. Eight participants took anticoagulants but did not differ from their age-matched peers in blood biomarkers and blood flow values. They were retained in the study. Key descriptive statistics of the sample are presented in Table 1.

\section{MRI data acquisition}

All images were acquired on a $3 \mathrm{~T}$ Verio scanner (Siemens $\mathrm{GmbH}$, Erlangen, Germany). The MR scanning protocol included conventional structural imaging and a comprehensive evaluation of the head and neck vasculature via 2D phase-contrast (PC) flow quantification. For brain volume estimation, the structural scans were acquired in the coronal plane using a 12-channel head coil. Structural imaging included an MP-RAGE sequence with the following parameters: echo time $(\mathrm{TE})=4.38 \mathrm{~ms}$, repetition time $(\mathrm{TR})=1600 \mathrm{~ms}$, inversion time $(\mathrm{TI})=800 \mathrm{~ms}$, field of view $(\mathrm{FOV})=256 \times 256 \mathrm{~mm}^{2}$, resolution $=.67 \times 0.67 \times 1.34$ $\mathrm{mm}^{3}$, matrix size $=384 \times 384$, flip angle $(\mathrm{FA})=8^{\circ}$, and GRAPPA factor $=2$. To evaluate possible white matter lesions, a set of 50 contiguous axial slices of fluid-attenuated inversion recovery (FLAIR) images was acquired with the following parameters: $\mathrm{TR}=8440 \mathrm{~ms}, \mathrm{TE}=112 \mathrm{~ms}$, $\mathrm{TI}=2200 \mathrm{~ms}, \mathrm{FA}=150^{\circ}, \mathrm{FOV}=256 \times 256 \mathrm{~mm}^{2}$, in-plane resolution $=1 \times 1 \mathrm{~mm}^{2}$, slice thickness $=2 \mathrm{~mm}$, and matrix size $=256 \times 256$. All MP-RAGE and FLAIR images were inspected for potential pathology, and possible incidental findings were reviewed by an experienced radiologist.

The PC-MRI images were acquired with a 16-channel head/neck coil perpendicular to the IJVs at the C2/ C3 level, which is superior to the carotid bifurcation and intersects the IJV inferior to the jugular foramen. The

Table 1 Sample descriptors

\begin{tabular}{llrr}
\hline & Range & Mean & \multicolumn{1}{c}{ SD } \\
\hline Age (years) & $18-74$ & 48.12 & 17.24 \\
Systolic blood pressure (mmHg) & $96.50-168.33$ & 119.90 & 13.55 \\
Diastolic blood pressure (mmHg) & $59.50-125.33$ & 75.96 & 9.96 \\
Hematocrit (\%) & $35.50-48.20$ & 41.70 & 2.99 \\
Exercise frequency (days per & $0-7$ & 3.40 & 2.12 \\
$\quad$ week) & & & \\
BMI & $17.17-38.22$ & 26.11 & 4.82 \\
Total brain volume $\left(\mathrm{cm}^{3}\right)$ & $895.48-1384.68$ & 1113.71 & 99.71 \\
Gray matter volume $\left(\mathrm{cm}^{3}\right)$ & $461.69-741.12$ & 580.43 & 59.76 \\
White matter volume $\left(\mathrm{cm}^{3}\right)$ & $426.13-650.00$ & 533.28 & 46.98 \\
MMSE & $26-30$ & 28.73 & 1.04 \\
Education (years) & $12-20$ & 15.37 & 1.88 \\
CES-D & $0-15$ & 5.11 & 4.38 \\
\hline
\end{tabular}

$B M I$ Body-Mass Index, MMSE Mini-Mental State Examination, CES$D$ Center for Epidemiologic Studies Depression Scale participant's head was secured with head coil restraints resting on the subject's forehead and a neck pillow positioned to the participant's comfort. The procedure produces internally consistent measures of venous and arterial blood flow (Sethi et al. 2015). After a brief neck vessel localizer scan, the PC-MRI sequence was acquired with the following parameters: $\mathrm{TR}=95.25 \mathrm{~ms}, \mathrm{TE}=10 \mathrm{~ms}, \mathrm{FA}=20^{\circ}$, FOV $=160 \times 160 \mathrm{~mm}^{2}$, in-plane resolution $=0.57 \times 0.57$ $\mathrm{mm}^{2}$, slice thickness $=2.5 \mathrm{~mm}$, matrix size $=448 \times 448$, bandwidth $=192 \mathrm{~Hz} / \mathrm{pix}$, GRAPPA $=2$, and maximum encoding velocity $($ VENC) $=50 \mathrm{~cm} / \mathrm{s}$ [see previous publications for details (Haacke et al. 2012, 2015; Feng et al. 2012)].

A note on parameter selection is in order. Higher VENC of $80 \mathrm{~cm} / \mathrm{s}$ has been used in some studies for measuring large vessel flow in the extracranial head and neck (e.g., Stoquart-Elsankari et al. 2009). However, we focused on venous flow, which tend to be slower especially in the paraspinal veins. Therefore, we opted for a lower VENC, which ensured higher signal-to-noise ratio (SNR) in the venous flow measurements, so that only one scan was needed. The tradeoff for improved SNR is possible aliasing of arterial vessel flow, which exceeds the VENC value. This tradeoff is mitigated if the peak velocity stays within a reasonable range (e.g., smaller than three times the VENC) and by applying the automatic unwrapping software (Lotz et al. 2002). In the SPIN software used in this study, we implemented a robust automatic unwrapping algorithm that compares pixelwise phase values in $\mathrm{x}, \mathrm{y}$, and $\mathrm{z}$ directions and ensures that only pixels that are aliased are unwrapped (Jiang et al. 2015). Moreover, in our experience, the venous flow aliasing is rare and a VENC value of $50 \mathrm{~cm} / \mathrm{s}$ is sufficiently high to handle it.

\section{MRI data processing}

The 2D PC-MRI images were processed with the inhouse written software, SPIN (Signal Processing in NMR, Detroit, MI, USA; http://mrinnovations.com/index. php?site=spin, last accessed 12/07/2015). The software was used for quantifying blood flow rate through major arteries and veins, including internal carotid arteries (ICA) and vertebral arteries (VA) for in-flow and the internal jugular veins (IJV) for outflow. Phase unwrapping was performed when the maximum velocity exceeded $50 \mathrm{~cm} / \mathrm{s}$. Two trained raters (A.M.D. and S.S.) delineated vessel boundaries on magnitude images in reference to the processed phase images using a computer-assisted full-width half-maximum (FWHM) region growing threshold method with manual editing when necessary (see Sethi et al. 2015 for details). In this manner, six vessels were evaluated: left and right IJV, ICA, and VA. Flow rates $(\mathrm{mL} / \mathrm{s})$ were calculated from differences in phase contrast over time, 
taking into account the vessel area for each left and right sides, and were summed over the two sides to calculate total flow rate. All flow measures had high inter-rater reliability estimated via intraclass correlations computed with an assumption of random raters [formula $\operatorname{ICC}(2,1)$; Shrout and Fleiss 1979]. The reliability for each vessel type was as follows: $\operatorname{IJV}, \operatorname{ICC}(2,1)=0.99$; $\operatorname{ICA} \operatorname{ICC}(2,1)=0.99$; VA, $\operatorname{ICC}(2,1)=0.93$; other smaller vessels (e.g., vertebral veins) could not be measured with comparably high reliability, and were thus omitted from analysis. For narrowing of the upper IJV body above $\mathrm{C} 3 / \mathrm{C} 4$, a cross-sectional area of less than $12.5 \mathrm{~mm}^{2}$ was considered stenotic (Haacke et al. 2015). Six participants met this criterion in at least one of their IJVs, and were, therefore, excluded from the analyses, as it was unclear if their flow was carried by collateral veins not measured in this study.

Total brain volumes, as well as volumes of the gray and white matter, were estimated from the MP-RAGE scans. The images were downsampled to $1 \mathrm{~mm}^{3}$ isotropic voxels and were processed with the FSL brain extraction tool (BET; Smith 2002) for total brain volume, as well as tissue segmentation with the FMRIB's automated segmentation tool (FAST; Zhang et al. 2001) for total gray and white matter volumes. To avoid overestimation, we used a value of 0.3 for the fractional intensity threshold option $(-\mathrm{f}$ in FSL) instead of the default 0.5 . To prevent overestimation, the mean partial volume fraction for gray and white matter was calculated using the fslstats option in FSL. The segmented images were then binarized, and the volume of each tissue type was determined. Finally, the mean partial volume fraction was multiplied by the volume of the binarized tissue regions to yield a volume for each tissue type.

\section{Genomic analysis}

The details of the genomic methods can be found in the previous publications (Raz et al. 2009, 2011). DNA was isolated from buccal cultures obtained in mouthwash samples, using a Gentra Autopure LS under the standard buccal cell protocol. After sequencing and purification, DNA was analyzed on an ABI PRISM 3700 DNA Analyzer. All 5 '-nuclease assays adapted from a quantitative PCR method (Lo et al. 2000) were performed on an Applied Biosystems 7900.

APOE (rs429358 and rs7412) polymorphisms were preamplified with forward 5'-CAATGCTACCGAGTTTTC TTCC- $3^{\prime}$ and reverse primers 5'-TTCAGATTCTTCACA GATGCGTA- $3^{\prime}$ in a $25 \mu \mathrm{l}$ reaction containing $2.5 \mathrm{mmol} / \mathrm{l}$ $\mathrm{MgCl} 2,0.5 \mu \mathrm{mol} / \mathrm{l}$ of the primers, $1.25 \mathrm{U}$ AmpliTaq Gold polymerase, and $200 \mu \mathrm{mol} / \mathrm{l} \mathrm{dATP}, \mathrm{dCTP}$, dGTP, and dTTP. The primers and probes for the rs 7412 assay were $5^{\prime}$-TCC GCGATGCCGATGAC-3', 5'-CCCCGGCCTGGTACAC3', VIC-CAGGCGCTTCTGC-NFQ, and FAM-CAGGCA
CTTCGC-NFQ. The primers and probes for the rs429358 assay were 5'-GCGGGCACGGCTGT-3'， 5'-GCTTGC GCAGGTGGGA-3', VIC-CATGGAGGACGTGTGCNFQ, and FAM-ATGGAGGACGTGCGC-NFQ. COMT val158met (rs4680) polymorphism was interrogated using Taqman SNP Genotyping assays. For ACE I/D (rs4646994) genotyping, DNA was amplified using either ACE-1721F (insertion) or ACE-1428F (deletion) and ACE-1826R and interrogated using the TaqMan probe 1745T.

The allelic groups of COMT val158met were distributed as follows: 20 val homozygotes; 11 met homozygotes; and 42 heterozygotes. Hardy-Weinberg equilibrium test: $\chi^{2}=$ 2.07, $p=.15$. In the distribution of ACE I/D alleles, heterozygotes constituted approximately half of the sample $(46 \%, N=36)$, with $25 \mathrm{D} / \mathrm{D}$ and $17 \mathrm{I} / \mathrm{I}$ homozygotes. The distribution of $A C E$ genotypes conformed to Hardy-Weinberg equilibrium: $\chi^{2}=0.35, p=.55$. For $A P O E$ polymorphism, the majority $(73 \%, N=53)$ were APOE 83 homozygotes, 5 were APOE 23 heterozygotes, 14 APOE 834 heterozygotes, and one APOE 44 homozygote. To avoid dealing with cells with $N<5$, the allelic groups were combined and the sample was divided into 15 (21\%) APOEE4 carriers and those who did not carry that allele $(N=58)$. Because the sample was heterogeneous with respect to the population of origin, we tested for differences in allele frequency between African-Americans (21\% of the sample) and European-Americans, and found no significant differences in allele distribution. For APOE $\varepsilon 4: \chi^{2}=0.01 p=.93$; COMT: $\chi^{2}=4.12, p=.13$, and ACE I/D, $\chi^{2}=1.68 p=.43$, the allele distributions of two populations did not differ.

\section{Statistical analyses}

A single multivariate general linear model was fitted to the data. In the full model, in-flow (via ICA and VA, combined) and outflow (via IJV) served as two levels of a within-subject variable. Because sex composition of the sample was unbalanced ( 45 women to 28 men, $\chi^{2}=3.959$; $p=.047)$, sex was included in the model as a categorical factor. Age (centered at its sample mean), total brain volumes, and vascular risk score were entered as continuous independent variables. Using brain volume as a covariate is preferred to taking a flow-to-brain volume ratio, as ratio variables have been demonstrated to be inferior to covariates or residualized variables for the purposes of correction (e.g., Arndt et al. 1991; Mathalon et al. 1993). For the analyses of genetic effects, the variants were dichotomized according to hypothetically "risky" alleles as two-level between-subject factors: ApoE ( 0 for no $\varepsilon 4$ allele vs. 1 for carrier of $\varepsilon 4)$, ACE ( 0 for I carriers vs. 1 for D homozygotes), and COMT (0 for met allele carriers vs. 1 for val homozygotes). Type III error was used to allow for unbalanced categorical groupings, and Huynh-Feldt correction 
was applied to correct for violation of sphericity assumption of the repeated measures analyses. The second-order interactions between pairs of independent variables were tested in a full model and if found non-significant $(p>.10)$, removed from the model; reduced model was evaluated afterwards and its results reported.

\section{Results}

\section{Descriptive statistics and preliminary analyses}

The full correlation matrix for the brain variables, age, and vascular risk is presented in Table 2. Inspection of that matrix indicates high correlations between the arterial and venous flow rates, but revealed no associations of flow indices with parenchymal volume. There was no association between age and the total brain, gray or white matter volumes. As mentioned above, hematocrit was unrelated to the blood flow rate, age, or vascular risk. On the other hand, the vascular risk index positively correlated with age and negatively related to arterial blood flow rate.

To assess the need for separating variables by left vs. right side, we conducted a preliminary analysis, which revealed greater blood flow rate in the right IJV compared to the left IJV $[5.16 \pm 2.16$ vs. $3.57 \pm 2.11 \mathrm{~mL} / \mathrm{s}$; $F(1,69)=16.96, p<.001]$, but there were no interactions between the vein side and age, sex, or vascular risk (all $F<1$, ns). ICA blood flow rate exhibited no lateral asymmetry: $F(1,69)=2.26, p=.14$, nor there were lateral differences in VA blood flow rate: $F<1$, ns. Interactions between the artery side and age, sex, or vascular risk were not significant (all $p>.20$ ). Therefore, to conserve degrees of freedom, for the main analyses, flow rate indicators were summed across right and left sides.

\section{Effects of age and vascular risk}

In the main analysis of the effects of age and vascular risk on blood flow rate, none of the interactions in the full model, except COMT $\times$ age, reached significance (all $p>.25$ ), and therefore, were removed from the model. Estimation of the reduced model revealed that in-flow was greater than outflow: $F(1,65)=15.76$, $p<.001 ; 10.28 \pm 0.22$ vs. $8.87 \pm 0.22 \mathrm{~mL} / \mathrm{s}$ (model adjusted means \pm standard errors here and throughout). The main effect of age $F(1,65)=9.44, p=.003$ reflected overall greater flow in the younger participants. The main effect of vascular risk $F(1,65)=4.03, p=.049$ was due to lower flow in persons with higher risk scores. Although age $\times$ vessel type and vascular risk $\times$ vessel type interactions were not significant, simple effects analysis revealed that both effects were significant only for the arterial, and not venous, flow. For age the effects were $F(1,65)=14.91, p<.001$ for arterial flow rate, and $F(1,65)=2.79, p=.10$ for venous flow rate. Bootstrap analysis with 5000 replacement samples of the whole sample produced regression coefficient estimates of $b=-0.03 \mathrm{~mL} / \mathrm{s} / \mathrm{year}$, with $95 \%$ confidence interval (CI) $-0.06 /-0.01$ for in-flow, and $b=-0.02,95 \% \mathrm{CI}$ $-0.05 / 0.02 \mathrm{~mL} / \mathrm{s} /$ year for outflow. For vascular risk, simple effects were as follows: for in-flow: $r=-.30, b=-0.68$, bootstrapped $95 \% \mathrm{CI}-1.25 /-0.20 \mathrm{~mL} / \mathrm{s} / \mathrm{unit}$ of risk, and for outflow: $r=-.19, b=-0.46,95 \% \mathrm{CI}-1.01$ to $0.14 \mathrm{~mL} / \mathrm{s} /$ unit of risk. Note that both bootstrap CIs for outflow included 0 . These analyses are illustrated with regression plots in Fig. 1.

A significant main effect of $A C E$ I/D: $F(1,65)=6.56$, $p=.01$, reflected lower flow in $\mathrm{D}$ homozygotes compared to the I carriers: $10.85 \pm 0.28$ vs. $9.73 \pm 0.38 \mathrm{~mL} / \mathrm{s}$ for in - flow and $9.53 \pm 0.35$ vs. $8.66 \pm 0.47 \mathrm{~mL} / \mathrm{s}$ for outflow. The main effect of $C O M T$ val158 met $[F(1,65)=12.61, p=.001]$ was

Table 2 Correlation matrix for blood flow, brain volume, vascular risk, and age

\begin{tabular}{|c|c|c|c|c|c|c|c|c|c|c|c|}
\hline & Age & Vascular risk & Brain & Gray & White & IJV flow & ICA flow & VA flow & BMI & Systolic & Diastolic \\
\hline Vascular risk & 0.411 & & & & & & & & & & \\
\hline Brain volume & -0.172 & 0.093 & & & & & & & & & \\
\hline Gray matter volume & -0.177 & 0.057 & 0.949 & & & & & & & & \\
\hline White matter volume & -0.140 & 0.124 & 0.916 & 0.741 & & & & & & & \\
\hline IJV flow & -0.122 & -0.185 & 0.027 & 0.045 & 0.000 & & & & & & \\
\hline ica flow & $-\underline{0.262}$ & -0.240 & 0.075 & 0.093 & 0.040 & 0.546 & & & & & \\
\hline va flow & -0.149 & -0.300 & 0.164 & 0.203 & 0.090 & 0.519 & 0.390 & & & & \\
\hline BMI & 0.182 & 0.624 & 0.092 & 0.055 & 0.126 & -0.010 & -0.300 & -0.222 & & & \\
\hline Systolic blood pressure & 0.406 & 0.940 & 0.077 & 0.044 & 0.107 & -0.162 & -0.202 & -0.262 & 0.592 & & \\
\hline Diastolic blood pressure & 0.163 & 0.747 & 0.171 & 0.144 & 0.179 & -0.159 & -0.097 & -0.226 & 0.559 & 0.811 & \\
\hline Hematocrit & -0.060 & 0.055 & 0.032 & -0.010 & 0.080 & 0.024 & 0.019 & -0.180 & 0.217 & 0.097 & 0.103 \\
\hline
\end{tabular}

Critical values: $r=.022, p \leq 0.05 ; r=0.27, p<.01 ; r=.35, \boldsymbol{p}<\mathbf{0 . 0 0 1}$

$B M I$ Body-Mass Index, $I J V$ internal jugular vein, $V A$ vertebral artery, $I C A$ internal carotid artery, $V R$ vascular risk score (see text for details) 


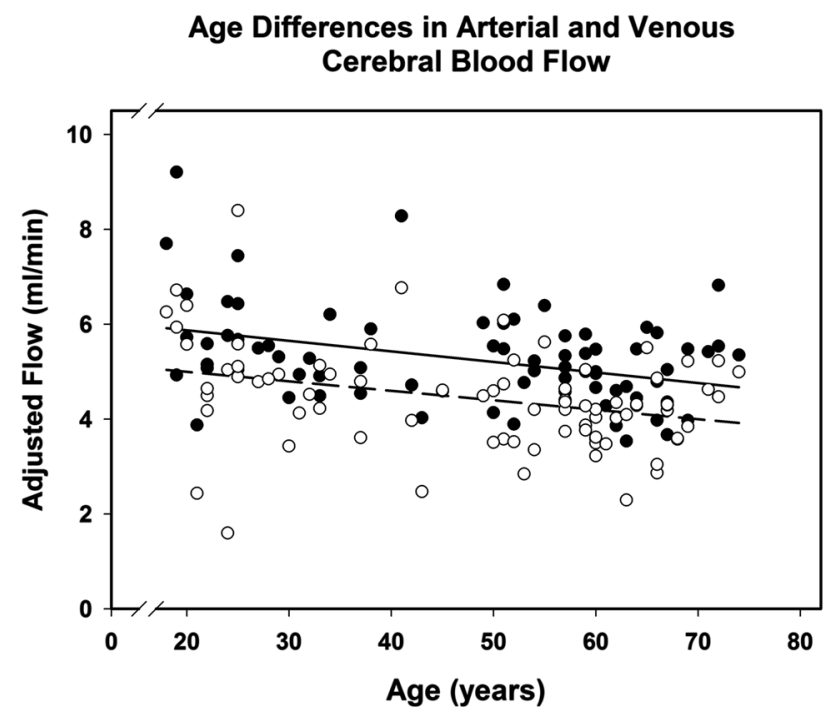

Arterial and Venous Cerebral Blood Flow and Vascular Risk

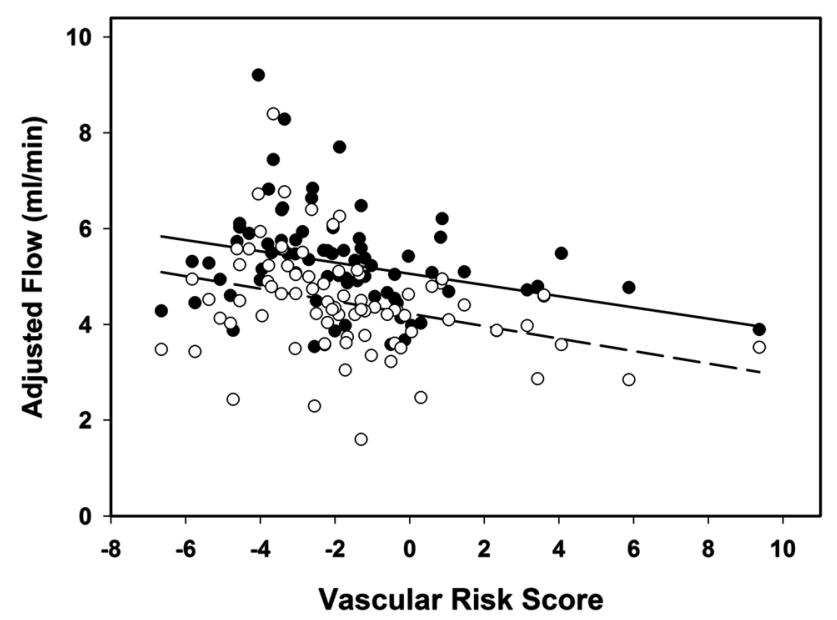

Fig. 1 Age- and vascular risk-related differences in the rate of cerebral blood flow: arterial (into the brain) and venous (out of the brain) due to carriers of the met variant having lesser blood flow rate in both directions: $10.75 \pm 0.40$ vs. $9.83 \pm 0.27 \mathrm{~mL} / \mathrm{s}$ for in-flow and $9.96 \pm 0.50$ vs. $8.23 \pm 0.33 \mathrm{~mL} / \mathrm{s}$ for outflow. The differences between allelic groups are illustrated in Fig. 2.

Notably, age differences were modified by a significant age $\times$ COMT interaction: $F(1,65)=14.76, p<.001$. Simple effects analyses with bootstrap estimates of regression coefficients indicated that age-related slowing of flow rate was observed only in COMT val homozygotes $(r=.72$; $b=-0.12 \mathrm{~mL} / \mathrm{s}$ per year, $95 \% \mathrm{CI}-0.16 / 0.06$ for in-flow and $r=.58, b=-0.09 \mathrm{~mL} / \mathrm{s}$ per year, $95 \% \mathrm{CI}-0.16 /-0.04$ for outflow) and not in met carriers $(r=-.10$ for in-flow and $r=.06$ for outflow, both ns). The age $\times$ COMT interaction is depicted in Fig. 3.

Neither sex differences nor $A P O E$ effect were noted: both $F<1$, ns. The addition of the brain volume as a covariate to the model did not affect the results except for moving the significance level for vascular risk from $p=.049$ to $p=.056$.

\section{Discussion}

The main novel finding in this study is that a genetic variant (ACE deletion) linked to increased propensity for vasoconstriction was associated with lower arterial and venous extra-cerebral flow. In addition, participants with higher composite load of vascular risk factors had lower extra-cerebral blood flow, although this association was more robust for in-flow than for outflow. Notably, a modest age-related reduction in arterial and venous flow was observed only in persons who did not carry the allele linked to greater availability of dopamine.

In contrast to reported higher arterial $\mathrm{CBF}$ in children who carried the met allele of COMT val158met (Thomason et al. 2009), we observed higher flow rates in adult val homozygotes. The observed interaction between genetic propensity for lesser dopamine availability and
Fig. 2 Differences in blood flow rate between aCOMT val158met val homozygotes and met carriers and $\mathbf{b}$ between $A C E$ $D$ homozygotes and $I$ carriers. The bars represent the leastsquare means from the general linear model (see text); the error bars are standard errors around the least-square means
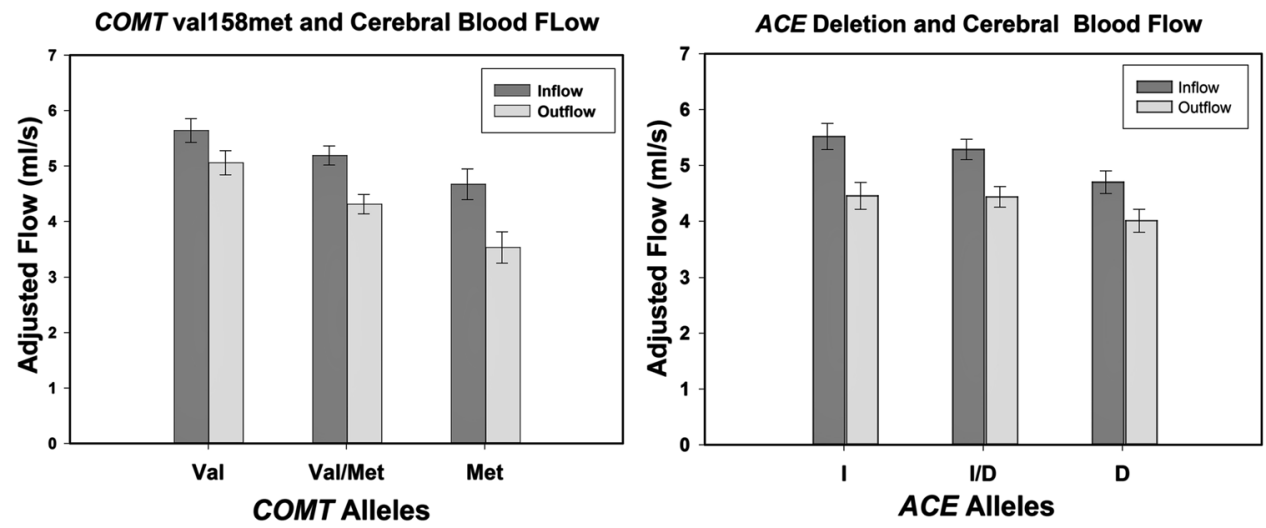


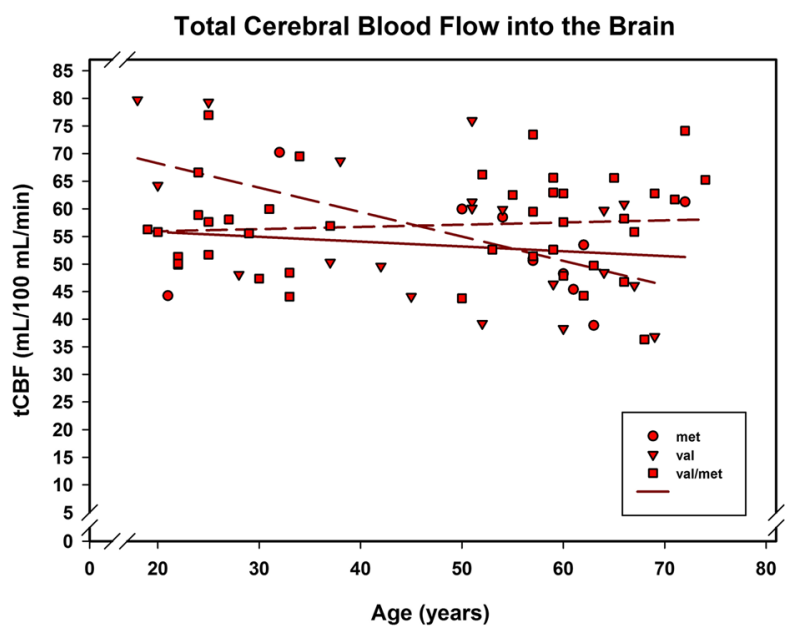

Total Cerebral Blood Flow out of the Brain

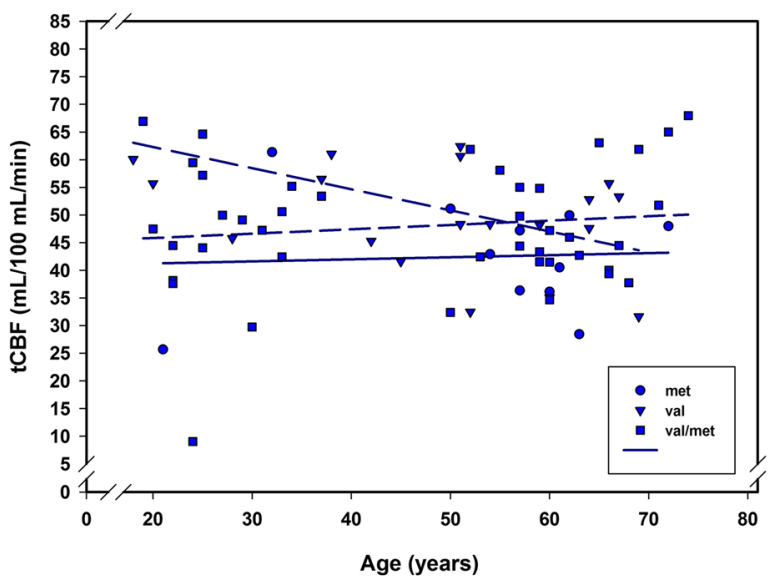

Fig. 3 Age-related differences in blood flow rate by COMT val158met genotype. The regression on age shows a significant negative slope only for val homozygotes (solid line) but not for met carriers (dashed line)

age differences in extra-cerebral blood flow rate mirrors the age-dopamine interaction in relation to cognitive performance as predicted by the inverted-U model (Bäckman et al. 2006, 2010). The inverted-U model posits that because of decline in dopamine in old age, the impact of genetic differences in dopamine availability on the brain and cognition increases with age. Persons who have lower amounts of dopamine due to genetic variation are more affected by age-related declines in the dopaminergic system than their counterparts who carry more dopamine-favorable genetic variants. Although the effects of genetic dopamine variation are not strong, they support the proposition that when it comes to aging, even the modest unfavorable genetic influence may provide a "nudge" in the direction of decline (Raz and Lustig 2014). It is possible that the effect of COMT val158 met on dopamine is pleiotropic with respect to age, i.e., that younger adult val homozygotes (lower available dopamine) evidence faster blood flow than met carriers, but children and older adults who have lower dopamine levels than young and middle age adults may have a particularly significant reduction in blood flow rate. The observed pattern of age and genetic differences in blood flow suggests a negative pleiotropism as observed in some previous studies that showed advantage for val/ val homozygotes of COMT val158met in younger age but not among older adults (e.g., Lee and Qui 2016). A larger population-based study covering the lifespan age range is needed to test this hypothesis.

The venous outflow was lesser than arterial in-flow, which suggests that some of the venous blood could have been drained through a system of collateral vessels. The role of vertebral and collateral drainage system in reducing age and vascular risk effects on the outflow compared to the inflow is a plausible hypothesis but with the current methods that restrict reliable measures to only major vessels, it could not be tested. Evidence to date, however, does not support a major role for collateral contributions to drainage in healthy adults (Zamboni et al. 2013). Although in persons with stenosis, collateral drainage is likely, exclusion of such participants from this study made this a remote possibility (Sethi et al. unpublished data). Moreover, in supine position as employed in our study, most of venous drainage occurs through the jugular vein (Shenkin et al. 1949).

Notably, in this sample, total brain size was unrelated to the blood flow rate and calendar age. Although age-related differences in parenchymal volume with age are observed in many studies ( $\operatorname{Raz} 2000$; Raz and Rodrigue 2006), this is not necessarily a universal phenomenon. This finding may stem from the fact that in our sample, the participants were selected for optimal health. Thus, the lack of brain volume associations with age and blood flow serves to underscore the sensitivity of blood flow rate to even moderate health risks in a highly-selected cohort.

The mechanisms of the observed age-related differences in arterial blood flow rate are unclear. Although total and regional $\mathrm{CBF}$ were linked to increase of transition time between arteries and arterioles as assessed by the arterial spin labeling (ASL) technique (Liu et al. 2012), it is unclear how comparable ASL findings are to our results that were obtained with PC-MRI. The latter produces higher estimates of $\mathrm{CBF}$ and is imperfectly correlated with the former (Dolui et al. 2016).

The results reported here may have important implications for interpreting age differences in task-related activation observed in fMRI studies. Although imaging based on brain oxygen-level difference (BOLD) effect is a staple of cognitive neuroscience of aging, interpretation of activation 
maps obtained in fMRI comparisons of young and older participants is not straightforward. BOLD is an essentially cerebrovascular phenomenon and its dependence on the amount and flow rate of arterial blood is crucial to understanding age differences in resting state, as well as taskrelated activation. With increase in vascular risk being an important characteristic of typical aging, and the observed association between physiological and genetic vascular risk factors with cerebral arterial blood flow rate, it is important to take age-related differences in blood flow into account when interpreting fMRI findings in age-heterogeneous samples. Recent research has shed some light on the relationship between CBF and the BOLD effect. The results of several investigations have shown that $\mathrm{CBF}$ response to cognitive load does not match changes in blood oxygen levels (Mohtasib et al. 2012), change in CBF does not support the required increase in oxygen metabolism during task performance (Hutchison et al. 2013) and lower baseline $\mathrm{CBF}$ is linked to reduced activation estimates in older adults (Ances et al. 2009).

The findings reported here may link individual differences in blood flow and BOLD through common genetic influence. Several characteristics of CBF are under substantial genetic control (Tarnoki et al. 2014), and so are the amplitude, latency, and duration of the hemodynamic response function (HRF), from which activation is inferred in fMRI (Shan et al. 2016). Identifying specific genetic variants that affect BOLD and CBF remains a task to be accomplished, although several candidate genes have been proposed. At the time of this writing, we have no knowledge about any studies of age differences in fMRI activation and ACE I/D variant. However, the literature on differences in BOLD that can be related to COMT val158met variant is quite sizeable, although somewhat contradictory (Papenberg et al. 2015).

In resting-state fMRI, COMT genotype modifies functional networks among brain regions, with low-dopamine, val homozygotes showing significantly lower functional connectivity in comparison with val/met heterozygotes and met homozygotes (Damoiseaux et al. 2016). In a sample of middle-aged and older adults, reduced task-related activation was noted in carriers of the val allele (Nyberg et al. 2014). Of note, in that study, participants with val/val genotype exhibited greater BOLD response on an easy task but not during demanding cognitive manipulation when their BOLD response was lower than that of the met carriers and in particular met/met homozygotes. Our finding of greater arterial flow rate in val/val homozygotes at rest makes lower resting flow in met/met homozygotes a plausible explanation of the reported differences in BOLD.

In comparing the results of this study to the extant literature, several characteristics should be taken into account. First, the participants were healthy volunteers who reported no history of cardiovascular or cerebrovascular diseases that are common on general population of older adults, particularly myocardial infarction, heart failure, or diabetes mellitus. Second, in this study, we restricted evaluation of the blood flow rate to extra-cerebral blood vessels, for which full detection and reliable measurements were confirmed and it is, therefore, unclear if the findings can be generalized to cerebral arteries and veins. Third, we examined hematocrit levels, which could affect blood flow velocity, and removed two participants with abnormally low values. We have thus eliminated blood viscosity as a potential confound, and in our sample, hematocrit was not associated with blood flow rate, total $\mathrm{CBF}$, age or vascular risk. Although we replicated the previous findings of right-sided dominance in IJV flow (Ayanzen et al. 2000; ElSankari et al. 2013; Stoquart-Elsankari et al. 2009), we observed no asymmetry in the arterial flow to the brain and the importance of lateral dominance in the flow rate remains unclear.

The results of this study should be interpreted in the context of several caveats and limitations. The most important one is that this is a cross-sectional study on a sample with a wide age range. It cannot, therefore, inform about age-related change or individual variability therein (Lindenberger et al. 2011).

Second, as all blood flow measures were conducted in a conventional MRI scanner, while the participants were in supine position, age differences in arterial flow could be underestimated, because delivery of arterial blood to the brain in this position requires lesser anti-gravity cardiac effort and may reduce the impact of age-related declines in cardiac output on flow rate.

Third, the lack of $A P O E \varepsilon 4$ effect may reflect a selection bias predicated on rigorous health screening and an almost total absence of $\varepsilon 4$ homozygotes. In contrast to APOE $\varepsilon 4$ and many other common genetic variants associated with health risks, $C O M T$ val158met and $A C E$ I/D display a relatively balanced distribution of alleles, regardless of the health status of the sample.

Fourth, a moderate sample size precluded examination of additional genetic variants, such as polymorphisms of the NOS gene nitric oxide synthase that may have a significant effect on CBF (Dalkara and Alarcon-Martinez 2015) and longevity in general (Montesanto et al. 2013).

Fifth, the effects of additional age-linked cardiovascular factors that have a profound effect on cerebral blood flow (e.g., reduction in cardiac output, Meng et al. 2015) should be examined in future studies in an attempt to explain agerelated variability in $\mathrm{CBF}$.

Sixth, a possible limitation and concern may be that the arterial-venous flow mismatch observed here is an artifact of averaging across the heart beat and respiration. This is unlikely for the following reasons. Flow from the entire cardiac cycle was collected in this study over a 
period of several minutes, so the cardiac data were averaged over many respiratory cycles. When measuring total flow, integrating the data over the cardiac cycle dramatically increases the SNR relative to a single timepoint measurement (Haacke and Patrick 1986; Nayak et al. 2015). Although the effect of respiration can cause a slight shift in the overall IJV flow $(\sim 2 \%)$ when a moving average over a given respiratory cycle is used (Billman 2011; Schrauben et al. 2015; Pucheu et al. 1994), the magnitude of this effect is unlikely to account for the observed arterial-venous mismatch.

Seventh, this study focused on IJV only because measurements of comparable reliability were not attained for the vertebral and smaller veins. Attention to rigorous reliability of venous flow assessment is important, especially in light of potentially misleading comparisons of vessels with differentially unreliable measures. Thus, future improvements in evaluation of venous flow in smaller vessels is important because of their measurable contribution to the outflow (Ciuti et al. 2013), although such measurements present an important challenge.

\section{Conclusion}

Even relatively minor elevation in vascular risk and propensity for greater vasoconstriction are linked to lower arterial blood flow and venous drainage rates. Age-related differences in flow rate were limited to a subgroup that was genetically predisposed to lesser synaptic dopamine availability. In contrast to stable and immutable genetic variations, vascular risk factors examined here can be modified and mitigated via life-style changes and pharmaceutical interventions (Yaffe et al. 2014). Thus, if indeed the differences in blood flow rate are linked to declines in cognitive performance, addressing such modifiable factors can be a valuable contribution to ameliorating the course of aging.

Acknowledgements This work was supported in part by a Grant from the National Institute on Aging R37 AG011230 to NR and National Heart, Lung and Blood Institute Grant R42 HL112580 to EMH. AMD was supported by a Beckman Institute Postdoctoral Fellowship at the University of Illinois at Urbana-Champaign, with funding provided by the Arnold and Mabel Beckman Foundation. The authors gratefully acknowledge Dr. Susan Land, Director of Wayne State University Applied Genomics Technology Center who conducted DNA analysis.

\section{References}

Aanerud J, Borghammer P, Chakravarty MM, Vang K, Rodell AB, Jónsdottir KY, Møller A, Ashkanian M, Vafaee MS, Iversen P, Johannsen P, Gjedde A (2012) Brain energy metabolism and blood flow differences in healthy aging. J Cereb Blood Flow Metab 32(7):1177-1187. doi:10.1038/jcbfm.2012.18

Ances BM, Liang CL, Leontiev O, Perthen JE, Fleisher AS, Lansing AE, Buxton RB (2009) Effects of aging on cerebral blood flow, oxygen metabolism, and blood oxygenation level dependent responses to visual stimulation. Hum Brain Mapp 30(4):1120-1132. doi:10.1002/hbm.20574

Armulik A, Genové G, Betsholtz C (2011) Pericytes: developmental, physiological, and pathological perspectives, problems, and promises. Dev Cell 21(2):193-215. doi:10.1016/j. devcel.2011.07.001

Arndt S, Cohen G, Alliger RJ, Swayze VW II, Andreasen NC (1991) Problems with ratio and proportion measures of imaged cerebral structures. Psychiatry Res Neuroimaging 40:79-89

Attwell D, Laughlin SB (2001) An energy budget for signaling in the grey matter of the brain. J Cereb Blood Flow Metab 21:1133-1145

Ayanzen RH, Bird CR, Keller PJ, McCully FJ, Theobald MR, Heiserman JE (2000) Cerebral MR venography: normal anatomy and potential diagnostic pitfalls. AJNR 21:74-78

Bäckman L, Nyberg L, Lindenberger U, Li SC, Farde L (2006) The correlative triad among aging, dopamine, and cognition: current status and future prospects. Neurosci Biobehav Rev 30(6):791-807

Bäckman L, Lindenberger U, Li SC, Nyberg L (2010) Linking cognitive aging to alterations in dopamine neurotransmitter functioning: recent data and future avenues. Neurosci Biobehav Rev 34(5):670-677. doi:10.1016/j.neubiorev.2009.12.008

Bautista LE, Vargas CI, Oróstegui M, Gamarra G (2008) Population-based case-control study of renin-angiotensin system genes polymorphisms and hypertension among Hispanics. Hypertens Res 31(3):401-408. doi:10.1291/hypres.31.401

Benetos A, Gautier S, Ricard S, Topouchian J, Asmar R, Poirier O, Larosa E, Guize L, Safar M, Soubrier F, Cambien F (1996) Influence of angiotensin-converting enzyme and angiotensin II type 1 receptor gene polymorphisms on aortic stiffness in normotensive and hypertensive patients. Circulation 94:698-703

Billman GE (2011) Heart rate variability-a historical perspective. Front Physiol 2:86. doi:10.3389/fphys.2011.00086. (eCollection 2011)

Casey CS, Atagi Y, Yamazaki Y, Shinohara M, Tachibana M, Fu Y, Bu G, Kanekiyo T (2015) Apolipoprotein E inhibits cerebrovascular pericyte mobility through a RhoA protein-mediated pathway. J Bio Chem 290(22):14208-14217. doi:10.1074/jbc. M114.625251(Epub 2015 Apr 22)

Chen JJ, Rosas HD, Salat DH (2011) Age-associated reductions in cerebral blood flow are independent from regional atrophy. Neuroimage 55:468-478. doi:10.1016/j. neuroimage.2010.12.032

Chung CP, Lin YJ, Chao AC, Lin SJ, Chen YY, Wang YJ, Hu HH (2010 Jugular venous hemodynamic changes with aging. Ultrasound Med Biol 36(11):1776-1782. doi:10.1016/j. ultrasmedbio.2010.07.006

Ciuti G, Righi D, Forzoni L, Fabbri A, Pignone AM (2013) Differences between internal jugular vein and vertebral vein flow examined in real time with the use of multigate ultrasound color Doppler. Am J Neuroradiol 34(10):2000-2004. doi:10.3174/ajnr. A3557(Epub 2013 May 30)

Dalkara T, Alarcon-Martinez L (2015) Cerebral microvascular pericytes and neurogliovascular signaling in health and disease. Brain Res 1623:3-17. doi:10.1016/j.brainres.2015.03.047(Epub 2015 Apr 8)

Damoiseaux JS, Viviano RP, Yuan P, Raz N (2016) Differential effect of age on posterior and anterior hippocampal functional connectivity. Neuroimage 133:468-476. doi:10.1016/j. neuroimage.2016.03.047(Epub 2016 Mar 28) 
Dixon RA, DeCarlo CA, MacDonald SWS, Vergote D, Jhamandas J and Westaway D (2014) APOE and COMT polymorphisms are complementary biomarkers of status, stability, and transitions in normal aging and early mild cognitive impairment. Front Aging Neurosci 6:236. doi:10.3389/fnagi.2014.00236

Doepp F, Schreiber SJ, von Münster T, Rademacher J, Klingebiel R, Valdueza JM (2004) How does the blood leave the brain? A systematic ultrasound analysis of cerebral venous drainage patterns. Neuroradiology 46(7):565-570

Dolui S, Wang Z, Wang DJ, Mattay R, Finkel M, Elliott M, Desiderio L, Inglis B, Mueller B, Stafford RB, Launer LJ, Jacobs DR Jr, Bryan RN, Detre JA (2016) Comparison of non-invasive MRI measurements of cerebral blood flow in a large multisite cohort. J Cereb Blood Flow Metab 36:1244-1256. doi:10.1177/0271678 X16646124(Epub 2016 May 3)

ElSankari S, Balédent O, van Pesch V, Christian Sindic C, de Broqueville Q, Duprez T (2013) Concomitant analysis of arterial, venous, and CSF flows using phase-contrast MRI: a quantitative comparison between MS patients and healthy controls. J Cer Blood Flow Metab 33:1314-1321. doi:10.1038/jcbfm.2013.95

Feng W, Utriainen D, Trifan G, Sethi S, Hubbard D, Haacke EM (2012) Quantitative flow measurements in the internal jugular veins of multiple sclerosis patients using magnetic resonance imaging. Rev Recent Clin Trials 7(2):117-126

Folstein MF, Folstein SE, McHugh PR (1975) "Mini-mental state": a practical method for grading the cognitive state of patients for the clinician. J Psychiatr Res 12(3):189-198

Greenfield JC Jr, Tindall GT (1968) Effect of norepinephrine, epinephrine, and angiotensin on blood flow in the internal carotid artery of man. J Clin Invest 47(7):1672-1684

Haacke EM, Patrick JL (1986) Reducing motion artifacts in twodimensional Fourier transform imaging. Magn Reson Imaging 4(4):359-376

Haacke EM, Feng W, Utriainen D, Trifan G, Wu Z, Latif Z, Katkuri Y, Hewett J, Hubbard D (2012) Patients with multiple sclerosis with structural venous abnormalities on MR imaging exhibit an abnormal flow distribution of the internal jugular veins. J Vasc Interv Radiol 23(1):60-68 .e1-e3. doi:10.1016/j.jvir.2011.09.027

Haacke EM, Sethi, SK, Ultriainen DT (2015) The role of magnetic resonance imaging in assessing venous vascular abnormalities in the head and neck: a demonstration of cerebrospinal venous insufficiency in a subset of multiple sclerosis patients. Veins Lymphat 4:5012

Hagen K, Pettersen E, Stovner LJ, Skorpen F, Holmen J, Zwart J-A (2007) High systolic blood pressure is associated with val/val genotype in the catechol- $O$-Methyltransferase gene. Am J Hyp 20:21-26

Hajjar I, Sorond F, Lipsitz LA (2015) Apolipoprotein E, carbon dioxide vasoreactivity, and cognition in older adults: effect of hypertension. J Am Ger Soc 63(2):276-281

Hall CN, Reynell C, Gesslein B, Hamilton NB, Mishra A, Sutherland BA, O'Farrell FM, Buchan AM, Lauritzen M, Attwell D (2014) Capillary pericytes regulate cerebral blood flow in health and disease. Nature 508(7494):55-60

Hassan A, Lansbury A, Catto AJ, Guthrie A, Spencer J, Craven C, Grant PJ, Bamford JM (2002) Angiotensin converting enzyme insertion/deletion genotype is associated with leukoaraiosis in lacunar syndromes. J Neurol Neurosurg Psychiatry 72(3):343-346

Henriksen OM, Jensen LT, Krabbe K, Guldberg P, Teerlink T, Rosturp E (2014) Resting brain perfusion and selected vascular risk factors in healthy elderly subjects. PLoS One 9(5):e97363. doi:10.1371/journal.pone.0097363

Hutchison JL, Lu H, Rypma B (2013) Neural mechanisms of age-related slowing: the $\triangle \mathrm{CBF} / \triangle \mathrm{CMRO} 2$ ratio mediates age-differences in BOLD signal and human performance. Cereb Cortex 23:2337-2346. doi:10.1093/cercor/bhs233(Epub 2012 Aug 8)

Jagust W (2013) Vulnerable neural systems and the borderland of brain aging and neurodegeneration. Neuron 77(2):219-234

Janciauskiene SM, Erikson C, Warkentin S (2009) A link between sICAM-1, ACE and parietal blood flow in the aging brain. Neurobiol Aging 30(9):1504-1511. doi:10.1016/j. neurobiolaging.2007.08.025(Epub 2008 Feb 19)

Jiang J, Kokeny P, Ying W, Magnano C, Zivadinov R, Mark Haacke E (2015) Quantifying errors in flow measurement using phase contrast magnetic resonance imaging: comparison of several boundary detection methods. Magn Reson Imaging 33(2):185-193. doi:10.1016/j.mri.2014.10.009(Epub 2014 Nov 12)

Jose PA, Eisner GM, Felder RA (2003) Regulation of blood pressure by dopamine receptors. Nephron Physiol 95(2):19-27

Kim SM, Kim MJ, Rhee HY, Ryu CW, Kim EJ, Petersen ET, Jahng GH (2013) Regional cerebral perfusion in patients with Alzheimer's disease and mild cognitive impairment: effect of APOE epsilon4 allele. Neuroradiology 55(1):25-34. doi:10.1007/ s00234-012-1077-x. (Epub 2012 Jul 25)

Lao JI, Montoriol C, Morer I, Beyer K (2005) Genetic contribution to aging: deleterious and helpful genes define life expectancy. Ann N Y Acad Sci 1057:50-63

Lee A, Qiu A (2016) Modulative effects of COMT haplotype on agerelated associations with brain morphology. Hum Brain Mapp. doi:10.1002/hbm.23161(Epub ahead of print)

Lindenberger U, von Oertzen T, Ghisletta P, Hertzog C (2011) Crosssectional age variance extraction: what's change got to do with it? Psychol Aging 26(1):34-47

Liu Y, Zhu X, Feinberg D, Guenther M, Gregori J, Weiner MW, Schuff N (2012) Arterial spin labeling MRI study of age and gender effects on brain perfusion hemodynamics. Magn Reson Med 68(3):912-922. doi:10.1002/mrm.23286(Epub 2011 Dec 2)

Lo YM, Lau TK, Chan LY, Leung TN, Chang AM (2000) Quantitative analysis of the bidirectional fetomaternal transfer of nucleated cells and plasma DNA. Clin Chem 46(9):1301-1309

Lotz J, Meier C, Leppert A, Galanski M (2002) Cardiovascular flow measurement with phase-contrast MR imaging: basic facts and implementation. Radiographics 22:651-671. doi:10.1148/radiog raphics.22.3.g02ma11651

Lynch AI, Arnett DK, Pankow JS, Miller MB, North KE, Eckfeldt JH, Hunt SC, Rao DC, Djoussé L (2007) Sex-specific effects of ACE I/D and AGT-M235T on pulse pressure: the HyperGEN Study. Hum Gen 122(1):33-40

Magnano C, Belov P, Krawiecki J, Hagemeier J, Beggs C, Zivadinov R (2016) Internal jugular vein cross-sectional area enlargement is associated with aging in healthy individuals. PLoS One 11(2):e0149532. doi:10.1371/journal.pone.0149532(eCollection 2016)

Mathalon DH, Sullivan EV, Rawles JM, Pfefferbaum A (1993) Correction for head size in brain-imaging measurement. Psychiatry Res Neuroimaging 50:121-139

Mattace-Raso FU, van der Cammen TJ, Sayed-Tabatabaei FA, van Popele NM, Asmar R, Schalekamp MA, Hofman A, van Duijn CM, Witteman JC (2004) Angiotensin-converting enzyme gene polymorphism and common carotid stiffness. The Rotterdam Study. Atherosclerosis 174:121-126

Meng L, Hou W, Chui J, Han R, Gelb AW (2015) Cardiac output and cerebral blood flow: the integrated regulation of brain perfusion in adult humans. Anesthesiology 123(5):1198-1208. doi:10.1097/ALN.0000000000000872

Messina EJ, Weiner R, Kaley G (1975) Inhibition of bradykinin vasodilation and potentiation of norepinephrine and angiotensin 
vasoconstriction by inhibitors of prostaglandin synthesis in skeletal muscle of the rat. Circ Res 37(4):430-437

Mohtasib RS, Lumley G, Goodwin JA, Emsley HC, Sluming V, Parkes LM (2012) Calibrated fMRI during a cognitive Stroop task reveals reduced metabolic response with increasing age. Neuroimage 59(2):1143-1151. doi:10.1016/j.neuroimage.2011.07.092

Montesanto A, Crocco P, Tallaro F, Pisani F, Mazzei B, Mari V, Corsonello A, Lattanzio F, Passarino G, Rose G (2013) Common polymorphisms in nitric oxide synthase (NOS) genes influence quality of aging and longevity in humans. Biogerontology 14(2):177-186. doi:10.1007/s10522-013-9421-z(Epub 2013 Apr 10)

Morgan DG, May PC, Finch CE (1987) Dopamine and serotonin systems in human and rodent brain: effects of age and neurodegenerative disease. J Am Ger Soc 35(4):334-345

Morra L, Zade D, McGlinchey RE, Milberg WP (2013) Normal aging and cognition: the unacknowledged contribution of cerebrovascular risk factors. Neuropsychol Dev Cog B Aging Neuropsychol Cog 20(3):271-297. doi:10.1080/13825585.2012.693905

Morris BJ, Zee RY, Schrader AP (1994) Different frequencies of angiotensin-converting enzyme genotypes in older hypertensive individuals. J Clin Invest 94:1085-1089

Mozaffarian D, Benjamin EJ, Go AS, Arnett DK, Blaha MJ, Cushman M, de Ferranti S, Després JP, Fullerton HJ, Howard VJ, Huffman MD, Judd SE, Kissela BM, Lackland DT, Lichtman JH, Lisabeth LD, Liu S, Mackey RH, Matchar DB, McGuire DK, Mohler ER 3rd, Moy CS, Muntner P, Mussolino ME, Nasir K, Neumar RW, Nichol G, Palaniappan L, Pandey DK, Reeves MJ, Rodriguez CJ, Sorlie PD, Stein J, Towfighi A, Turan TN, Virani SS, Willey JZ, Woo D, Yeh RW, Turner MB; American Heart Association Statistics Committee and Stroke Statistics Subcommittee (2015) Heart disease and stroke statistics-2015 update: a report from the American Heart Association. Circulation 131(4): e29-322. doi:10.1161/CIR.0000000000000152. (Epub 2014 Dec 17)

Nayak KS, Nielsen JF, Bernstein MA, Markl M, D Gatehouse P, M Botnar R, Saloner D, Lorenz C, Wen H, S Hu B, Epstein FH, N Oshinski J, Raman SV (2015) Cardiovascular magnetic resonance phase contrast imaging. J Cardiovasc Magn Reson 17:71. doi:10.1186/s12968-015-0172-7

Nishijima Y, Akamatsu Y, Weinstein PR, Liu J (2015) Collaterals: implications in cerebral ischemic diseases and therapeutic interventions. Brain Res 623:18-29. doi:10.1016/j. brainres.2015.03.006(Epub 2015 Mar 11)

Nyberg L, Andersson M, Kauppi K, Lundquist A, Persson J, Pudas S, Nilsson LG (2014) Age-related and genetic modulation of frontal cortex efficiency. J Cogn Neurosci 26(4):746-754. doi:10.1162/ jocn_a_00521(Epub 2013 Nov 18)

Papenberg G, Salami A, Persson J, Lindenberger U, Bäckman L (2015) Genetics and functional imaging: effects of APOE, BDNF, COMT, and KIBRA in aging. Neuropsychol Rev 25(1):47-62. doi:10.1007/s11065-015-9279-8(Epub 2015 Feb 10)

Parkes LM, Rashid W, Chard DT, Tofts PS (2004) Normal cerebral perfusion measurements using arterial spin labeling: reproducibility, stability, and age and gender effects. Magn Reson Med 51:736-743. doi:10.1002/mrm.20023

Pucheu A, Evans J, Thomas D, Scheuble C, Pucheu M (1994) Doppler ultrasonography of normal neck veins. J Clin Ultrasound 22(6):367-373

Raz N (2000) Aging of the brain and its impact on cognitive performance: integration of structural and functional findings. In: Craik FIM, Salthouse TA (eds) Handbook of aging and cognition-II. Erlbaum, Mahwah, pp 1-90

Raz N, Lustig C (2014) Genetic variants and cognitive aging: destiny or a nudge? Psychol Aging 29(2):359-362. doi:10.1037/ a0036893
Raz N, Rodrigue KM (2006) Differential aging of the brain: Patterns, cognitive correlates and modifiers. Neurosci Biobehav Rev 30:730-748

Raz N, Rodrigue KM, Kennedy KM, Land S (2009) Genetic and vascular modifiers of age-sensitive cognitive skills: effects of COMT, BDNF, ApoE and hypertension. Neuropsychology 23:105-116

Raz N, Dahle CL, Rodrigue KM, Kennedy KM, Land S (2011) Effects of age, genes, and pulse pressure on executive functions in healthy adults. Neurobiol Aging 32:1124-1137

Saidi S, Zammiti W, Slamia LB, Ammou SB, Almawi WY, Mahjoub $\mathrm{T}$ (2009) Interaction of angiotensin-converting enzyme and apolipoprotein $\mathrm{E}$ gene polymorphisms in ischemic stroke involving large-vessel disease. J Thromb Thromb 27(1):68-74

Schrauben EM, Anderson AG, Johnson KM, Wieben O (2015) Respiratory-induced venous blood flow effects using flexible retrospective double-gating. J Magn Reson Imaging 42(1):211-216. doi:10.1002/jmri.24746(Epub 2014 Sep 11)

Sethi SK, Utriainen D, Daugherty AM, Feng W, Hewett JJ, Raz N, Haacke EM (2015) Jugular venous flow abnormalities in multiple sclerosis patients compared to normal controls. J Neuroimaging 25(4):600-607. doi:10.1111/jon.12183(Epub 2014 Oct 15)

Shan ZY, Vinkhuyzen AA, Thompson PM, McMahon KL, Blokland GA, de Zubicaray GI, Calhoun V, Martin NG, Visscher PM, Wright MJ, Reutens DC (2016). Genes influence the amplitude and timing of brain hemodynamic responses. NeuroImage $124(\mathrm{Pt} \quad \mathrm{A}): 663-671$. doi:10.1016/j.neuroimage.2015.09.016. (Epub 2015 Sep 12)

Shenkin HA, Scheuerman WG, Spitz EB, Groff RA (1949) The effects of change of position upon the cerebral circulation of man. J Appl Physiol 2(6):317-326

Shirahata N, Henriksen L, Vorstrup S, Holm S, Lauritzen M, Paulson OB, Lassen NA (1985) Regional cerebral blood flow assessed by $133 \mathrm{Xe}$ inhalation and emission tomography: normal values. J Comput Assist Tomogr 9(5):861-866

Shrout PE, Fleiss JL (1979) Intraclass correlations: uses in assessing rater reliability. Psychol Bull 86(2):420-428

Smith SM (2002) Fast robust automated brain extraction. Hum Brain Mapp 17(3):143-155

Song Y, Stampfer MJ, Liu S (2004) Meta-analysis: apolipoprotein E genotypes and risk for coronary heart disease. Annals Int Med 141:137-147

Stoquart-Elsankari S, Lehmann P, Villette A, Czosnyka M, Meyer ME, Deramond H, Balédent O (2009) A phase-contrast MRI study of physiologic cerebral venous flow. J Cereb Blood Flow Metab 29:1208-1215. doi:10.1038/sj.jcbfm.jcbfm200929

Taniwaki H, Kawagishi T, Emoto M, Shoji T, Hosoi M, Kogawa K, Nishizawa Y, Morii H (1999) Association of ACE gene polymorphism with arterial stiffness in patients with type 2 diabetes. Diabetes Care 22:1858-1864

Tarnoki AD, Tarnoki DL, Giannoni MF, Baracchini C, Meneghetti G, Cardaioli G, Medda E, Stazi MA, Cotichini R, Fagnani C, Nisticò L, Lucatelli P, Fanelli F, Berczi V, Garami Z, Littvay L, Schillaci G (2014) Heritability of cerebral arterial velocity and resistance. J Cardiovasc Med (Hagerstown) (Epub ahead of print)

ten Dam VH, van den Heuvel DM, de Craen AJ, Bollen EL, Murray $\mathrm{HM}$, Westendorp RG, Blauw GJ, van Buchem MA (2007) Decline in total cerebral blood flow is linked with increase in periventricular but not deep white matter hyperintensities. Radiology 243(1):198-203

Thambisetty M, Beason-Held L, An Y, Kraut MA, Resnick SM (2010) APOE $\varepsilon 4$ genotype and longitudinal changes in cerebral blood flow in normal aging. Arch Neurol 67(1):93-98 
Thomason ME, Waugh CE, Glover GH, Gotlib IH (2009) COMT genotype and resting brain perfusion in children. NeuroImage 48(1):217-222

van Es AC, van der Grond J, ten Dam VH, de Craen AJ, Blauw GJ, Westendorp RG, Admiraal-Behloul F, van Buchem MA; PROSPER Study Group (2010). Associations between total cerebral blood flow and age related changes of the brain. PLoS One 5(3):e9825. doi:10.1371/journal.pone.0009825

Vauquelin G, Michotte Y, Smolders I, Sarre S, Ebinger G, Dupont A, Vanderheyden P (2002) Cellular targets for angiotensin II fragments: pharmacological and molecular evidence. J Renin Angiotensin Aldosterone Syst 3(4):195-204

Vernooij MW, van der Lugt A, Ikram MA, Wielopolski PA, Vrooman HA, Hofman A, Krestin GP, Breteler MMB (2008) Total cerebral blood flow and total brain perfusion in the general population: the Rotterdam Scan Study. J Cereb Blood Flow Metab 28:412-419

von Bohlen und Halbach O, Albrecht D (2006) The CNS renin-angiotensin system. Cell Tiss Res 326(2):599-616. doi:10.1007/ s00441-006-0190-8

Yaffe K, Hoang TD, Byers AL, Barnes DE, Friedl KE (2014) Lifestyle and health-related risk factors and risk of cognitive aging among older veterans. Alzheimers Dement 10(3 Suppl):S111-121. doi:10.1016/j.jalz.2014.04.010
Zamboni P, Sisini F, Menegatti E, Taibi A, Malagoni AM, Morovic S, Gambaccini M (2013) An ultrasound model to calculate the brain blood outflow through collateral vessels: a pilot study. BMC Neurol 13:81. doi:10.1186/1471-2377-13-81

Zarrinkoob L, Ambarki K, Wåhlin A, Birgander R, Eklund A, Malm J (2015) Blood flow distribution in cerebral arteries. J Cereb Blood Flow Metab 35(4):648-654. doi:10.1038/jcbfm.2014.241

Zeng C, Zhang M, Asico LD, Eisner GM, Jose PA (2007) The dopaminergic system in hypertension. Clin Sci (Lond) 112(12):583-597

Zhang Y, Brady M, Smith S (2001) Segmentation of brain MR images through a hidden Markov random field model and the expectation-maximization algorithm. IEEE Trans Med Imag 20(1):45-57

Zhao M, Amin-Hanjani S, Ruland S, Curcio AP, Ostergren L, Charbel FT (2007) Regional cerebral blood flow using quantitative MR angiography. AJNR 28(8):1470-1473

Zhu S, Wang Z, Wu X, Shu Y, Lu D (2014) Apolipoprotein E polymorphism is associated with lower extremity deep venous thrombosis: color-flow Doppler ultrasound evaluation. Lipids Health Dis 13:21

Zivadinov R, Chung CP (2013) Potential involvement of the extracranial venous system in central nervous system disorders and aging. BMC Med 11:260. doi:10.1186/1741-7015-11-260 